\title{
Phase transitions in thermally annealed films of $\mathrm{Alq}_{3}$
}

\author{
G. Baldacchini \\ Via Guglielmo Quattrucci 246, 00046 Grottaferrata, Roma, Italy \\ E-mail: giuseppe.baldacchini@enea.it \\ giuseppe.baldacchini@gmail.com \\ P. Chiacchiaretta \\ Department of Neuroscience and Imaging, University G. D'Annunzio, 66013 Chieti, Italy \\ R.B. Pode \\ Department of Physics, Kyung Hee University, 1 Hoegi-dong, Dongdaemun-gu, Seoul 130-701, South Korea
}

\section{M.A. Vincenti}

ENEA, Photonics Micro- and Nano-structures Laboratory, UTAPRAD-MNF,

C.R. Frascati, Via E. Fermi 45, 00044 Frascati, Rome, Italy

\section{Q.-M. Wang}

School of Chemistry and Environment, South China Normal University, Waihuan Xi Road 378,

Guangzhou 510006, P. R. China 510006

Received April 12, 2012

\begin{abstract}
Organic light emitting devices have been so very much improved lately that they are being widely applied for displays and lighting. Among many improving technologies, annealing processes in different atmospheres affect greatly their performance, for instance when the active material is the small molecule $\mathrm{Alq}_{3}$. In particular, a significant increase of the photoluminescence is observed in thin films of this molecule between 150 and $180{ }^{\circ} \mathrm{C}$, before the physical destruction of the films occurs at higher temperatures. This phenomenon is attributed to a phase transition towards a novel morphological aggregation of the molecules in the film, which proved to be a much improved optical material for luminescent applications, and which seems common to other molecules as well.
\end{abstract}

PACS: 78.40.Me Organic compounds and polymers;

78.55.Kz Solid organic materials;

78.66.Qn Polymers; organic compounds;

81.30.Hd Constant-composition solid-solid phase transformations: polymorphic, massive, and order-disorder;

81.40.Ef Cold working, work hardening; annealing, post-deformation annealing, quenching, tempering recovery, and crystallization.

Keywords: $\mathrm{Alq}_{3}$, thin films, spectroscopy absorption, spectroscopy emission, phase transitions.

\section{Introduction}

The pioneering work of Tang and Van Slyke [1] aroused much interest in the $\mathrm{Alq}_{3}$ molecule, tris(8-hydroxyquinoline)aluminum, for its promising applications in optoelectronics [2]. As a consequence, systematic studies of optical, spectroscopic and structural properties on this organometallic compound, and other optical active organic materials as well, were systematically performed in order to clarify the fascinating chemistry and physics involved with them $[3,4]$.

Among many technical approaches, thermal annealing was discovered to be very effective in influencing the above properties. As a matter of facts, it was found that $\mathrm{Alq}_{3}$ films display a sizeable variation of the intensity, peak wavelength, and linewidth of the emission band as a 
function of temperature up to $200{ }^{\circ} \mathrm{C}[5,6]$. The particular behavior of such results suggested that they may have been originated by phase transition phenomena, which at their completion improved consistently the optical and morphological properties of the material, increasing so the general interest in $\mathrm{Alq}_{3}$ as a crucial component of organic light emitting devices (OLEDs).

However, it was clear since the beginnings of these studies that such behaviors could not be limited only to the $\mathrm{Alq}_{3}$ molecule, but they should have been rather common among other organometallic molecules in particular, and organic compounds in general. Indeed, many such materials possess similar chemical bonds and display more or less the same intermolecular interactions, so that some properties are also expected to be rather shared among them. In fact, recently there have been a few prominent reports about such similarities.

A slightly different molecule, as $\mathrm{Znq}_{2}$, displays a stable form having strong blue photoluminescence (PL) after ageing and annealing in various atmospheres, and there is a structural transformation in $\mathrm{Znq}_{2}$ films which gives rise to large changes in their optical properties [7]. Films of $\mathrm{Gaq}_{3}$ annealed in nitrogen gas show an enhanced absorption and emission with respect to the pristine film, and also a blue shift of the emission band has been observed [8]. These experimental results are similar in several aspects to our previous ones $[5,6]$ and to other ones never fully published until now, so that it deemed necessary to proceed to a detailed and systematic description of the experiments performed in $\mathrm{Alq}_{3}$ films, in order to perform a meaningful comparative study. Only in this way it will be possible to understand what is going on at level of molecular interactions and to attempt, if possible, an extension of the obtained results to many other organic compounds, having in mind both basic properties of optical active materials and applications in OLEDs.

\section{Experimental details and measurements}

Pure $\mathrm{Alq}_{3}$ films were grown on glass substrates by thermal evaporation. The starting $\mathrm{Alq}_{3}$ powder, $99.995 \%$ pure, obtained from Aldrich Company, was used without any further purification. The substrates were kept at room temperature (RT) on a copper holder, and the vacuum pressure in the evaporation chamber was about $10^{-6}$ Torr. The distance of $28 \mathrm{~cm}$ between the crucibles and substrates has been used for the majority of the evaporations. The films were grown at a rate $\sim 1 \mathrm{~nm} / \mathrm{min}$ and their thickness measured with a Tencor Alphastep Profilometer.

The fluorescence spectra were measured in open atmosphere at RT by using a Jobin Yvon Fluorolog-3 Spectrofluorometer with the front-face detecting geometry, and by exciting the samples at $395 \mathrm{~nm}$. Also the absorption spectra have been measured every so often with a PerkinElmer $\lambda$-19 Spectrophotometer. The main measurements reported here were performed on twelve samples, which were individually annealed at different temperatures and atmospheres, as described in Table 1. After the initial thermal treatment, the samples were kept in the atmosphere of the laboratory, where the extreme environment conditions were $15-25^{\circ} \mathrm{C}$ and $40-60 \%$ humidity in winter-summer, while optical emission and absorption have been measured during six years on a regular schedule. However, in the present work only the initial optical properties during the annealing treatments will be considered in detail, because of their direct connection with the supposed phase transition, while the optical properties during the long time of experimental observation will be only hinted here and fully described elsewhere.

Figure 1 shows the absorption spectrum of sample alq63-1 measured at RT and during the annealing process in dry nitrogen at 80,130 and $180^{\circ} \mathrm{C}$. The spectra are typical of $\mathrm{Alq}_{3}$ films with two absorption bands peaked at about 275 and $400 \mathrm{~nm}$. It is immediately observed that temperatures up to $130{ }^{\circ} \mathrm{C}$ do not affect the absorption, while at $180{ }^{\circ} \mathrm{C}$ there is a noticeable variation in the wavelength range $270-420 \mathrm{~nm}$, especially pronounced in the vicinity of $300 \mathrm{~nm}$ where the absorption increases more. Figure 2 shows the absorption spectrum of sample alq64-3 measured at RT and during the annealing process in wet nitrogen at 80 and $180^{\circ} \mathrm{C}$. As in the previous case, there are not variations at $80^{\circ} \mathrm{C}$, but the whole absorption de-

Table 1. $\mathrm{Alq}_{3}$ films annealed for $10 \mathrm{~min}$ in different atmospheres and temperatures as indicated: $a$ and $n$ stands for the sample still optically active or non-active after the last annealing process, respectively. The sample alq63-3 has not been annealed and is considered the reference sample

\begin{tabular}{c|c|c|c|c|c|c|c|c|c|c|c|c}
\hline \hline Batch & \multicolumn{4}{|c|}{ alq63 } & \multicolumn{3}{c|}{ alq64 } & \multicolumn{3}{c}{ alq65 } \\
\hline \hline Sample & 1 & 2 & 3 & 4 & 1 & 2 & 3 & 4 & 1 & 2 & 3 & 4 \\
\hline Thickness, nm & 45 & 45 & 45 & 45 & 55 & 55 & 55 & 55 & 55 & 55 & 55 & 55 \\
\hline Annealing & 80 & 80 & none & 80 & 80 & 88 & 80 & 80 & 180 & 155 & 180 & 145 \\
temperature, ${ }^{\circ} \mathrm{C}$ & 130 & 180 & & 180 & 144 & 155 & 180 & 160 & & & \\
& 180 & 250 & & 250 & & & & & & & \\
\hline Annealing & dry & dry & \multirow{2}{*}{ none } & dry & wet & wet & wet & wet & dry & wet & dry & wet \\
atmosphere & $\mathrm{N}_{2}$ & $\mathrm{O}_{2}$ & & $\mathrm{~N}_{2}$ & $\mathrm{~N}_{2}$ & $\mathrm{O}_{2}$ & $\mathrm{~N}_{2}$ & Air & $\mathrm{O}_{2}$ & $\mathrm{O}_{2}$ & $\mathrm{~N}_{2}$ & $\mathrm{~N}_{2}$ \\
\hline Final status & $a$ & $a$ & $a$ & $n$ & $a$ & $a$ & $n$ & $a$ & $a$ & $a$ & $a$ & $a$ \\
\hline
\end{tabular}




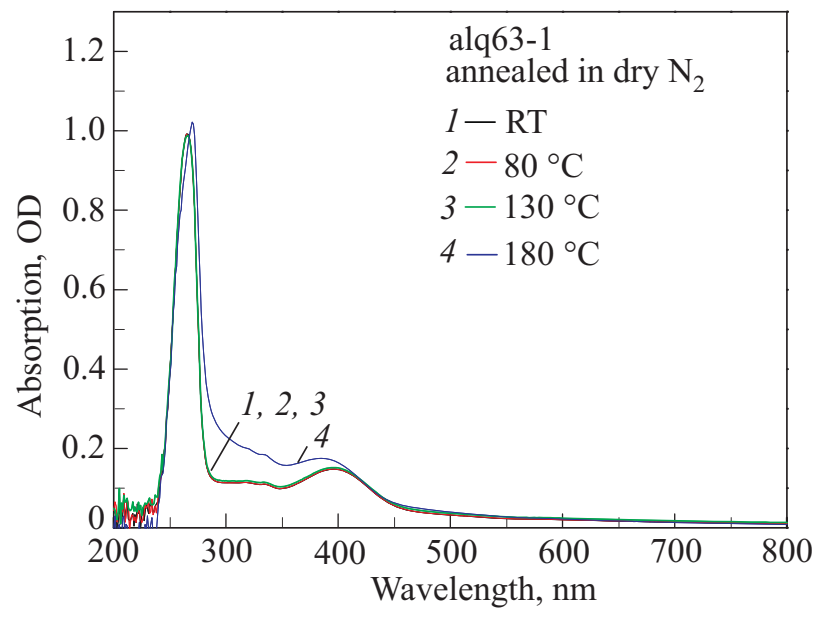

Fig. 1. (Color online) Absorption bands of sample alq63-1 during annealing treatment under dry nitrogen atmosphere. The sharp cutting below $240 \mathrm{~nm}$ is due to the absorption of the glass substrate.

creases consistently at $180{ }^{\circ} \mathrm{C}$. In order to understand the behavior of $\mathrm{Alq}_{3}$ films in wet atmospheres at intermediate temperatures, a measurement was performed at $145^{\circ} \mathrm{C}$ in sample alq65-4, and Fig. 3 shows the results. As in the case of dry nitrogen, also in wet nitrogen there is an increase of the absorption in the range $270-420 \mathrm{~nm}$, but less pronounced. Anyway, the previous results show the dominant role of temperature during the annealing process, but also disclose the strong reactive action of water which decreases to a trickle the absorption of sample alq64-3 at $180{ }^{\circ} \mathrm{C}$, see Fig. 2, while sample alq63-1 at the same temperature, but without water, displays a whole absorption, see Fig. 1.

It is well known that light pumping at any wavelength shorter than $450 \mathrm{~nm}$ produces an intense greenish luminescence, and here it has been chosen to pump at $395 \mathrm{~nm}$ at the maximum of the weakest absorption band. The emission bands of sample alq63-1 at each annealing temperature are shown in Fig. 4 and, as in the absorption case, no

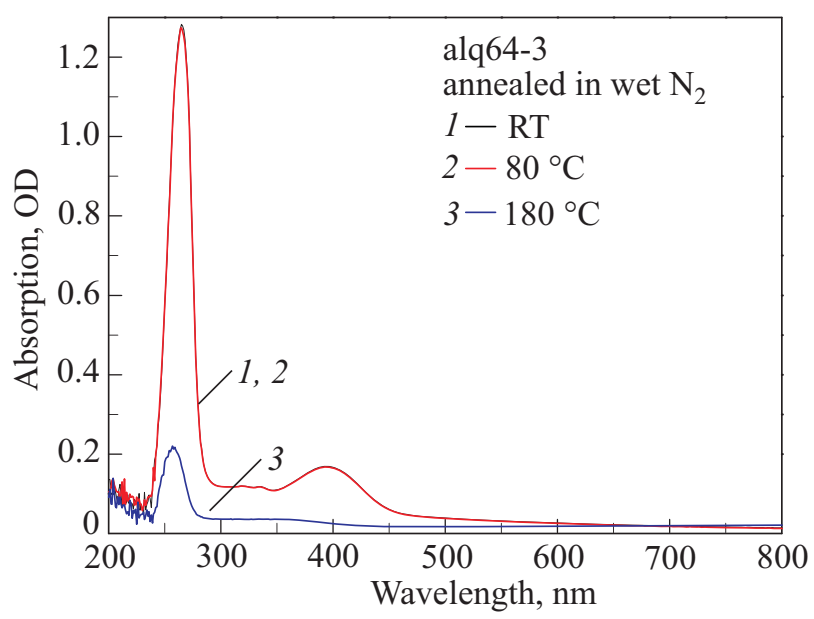

Fig. 2. (Color online) Absorption bands of sample alq64-3 during annealing treatment under wet nitrogen atmosphere. The sharp cutting below $240 \mathrm{~nm}$ is due to the absorption of the glass substrate.

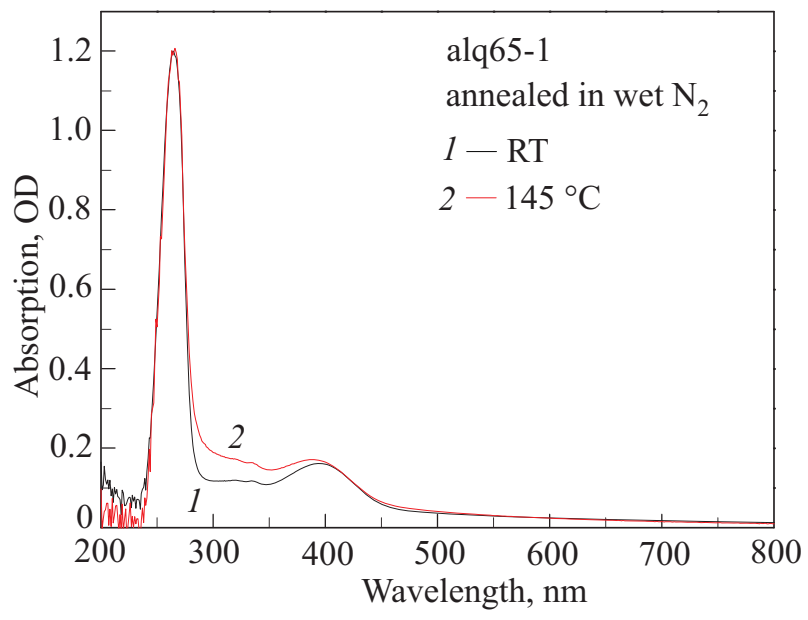

Fig. 3. (Color online) Absorption bands of sample alq65-4 during annealing treatment under wet nitrogen atmosphere. The sharp cutting below $240 \mathrm{~nm}$ is due to the absorption of the glass substrate.

variation is observed up to $130{ }^{\circ} \mathrm{C}$, while a consistent increase of the emission intensity at $180^{\circ} \mathrm{C}$ is evident, with also a noticeable blue shift. Less observable, there is also a shape variation in the emission band. Similar results are observed in Fig. 5 which refers to samples annealed in wet nitrogen atmospheres alq64-3 and alq65-4. However, practically no emission is observed in sample alq64-3 at $180^{\circ} \mathrm{C}$, which agrees with its corresponding very small absorption, and there is an increase of emission in sample alq65-4 which is slightly less than in the case of dry atmosphere of sample alq63-1. Again, the strong reaction of water on the $\mathrm{Alq}_{3}$ molecule at high temperatures is disclosed.

By looking at the previous experimental results, it is immediately seen that the emission intensity increases by about a factor 3 after annealing between 140 and $180{ }^{\circ} \mathrm{C}$, irrespectively from the environmental atmosphere. At the same time the absorption at $395 \mathrm{~nm}$ does not change at all in sample alq65-4 or increases by about $20 \%$ in sample alq63-1. So, there is not correspondence between the inten-

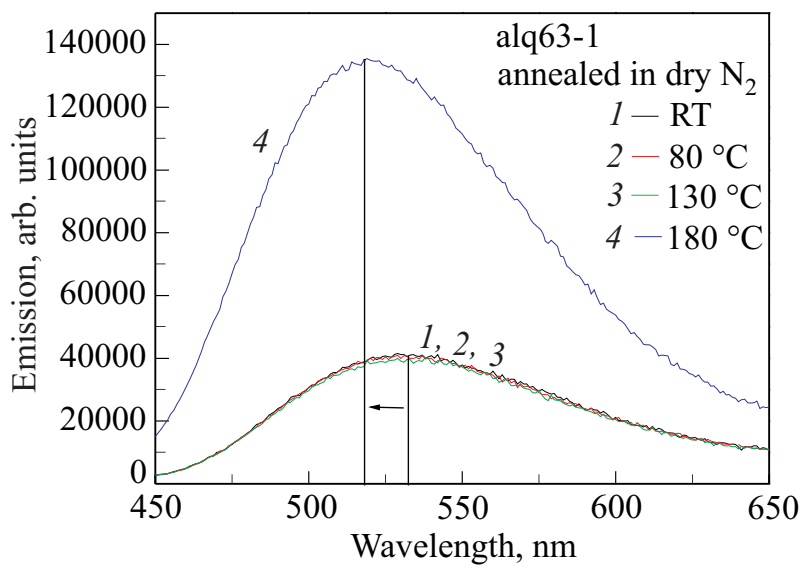

Fig. 4. (Color online) Emission bands of sample alq63-1 during annealing treatment under dry nitrogen atmosphere. 

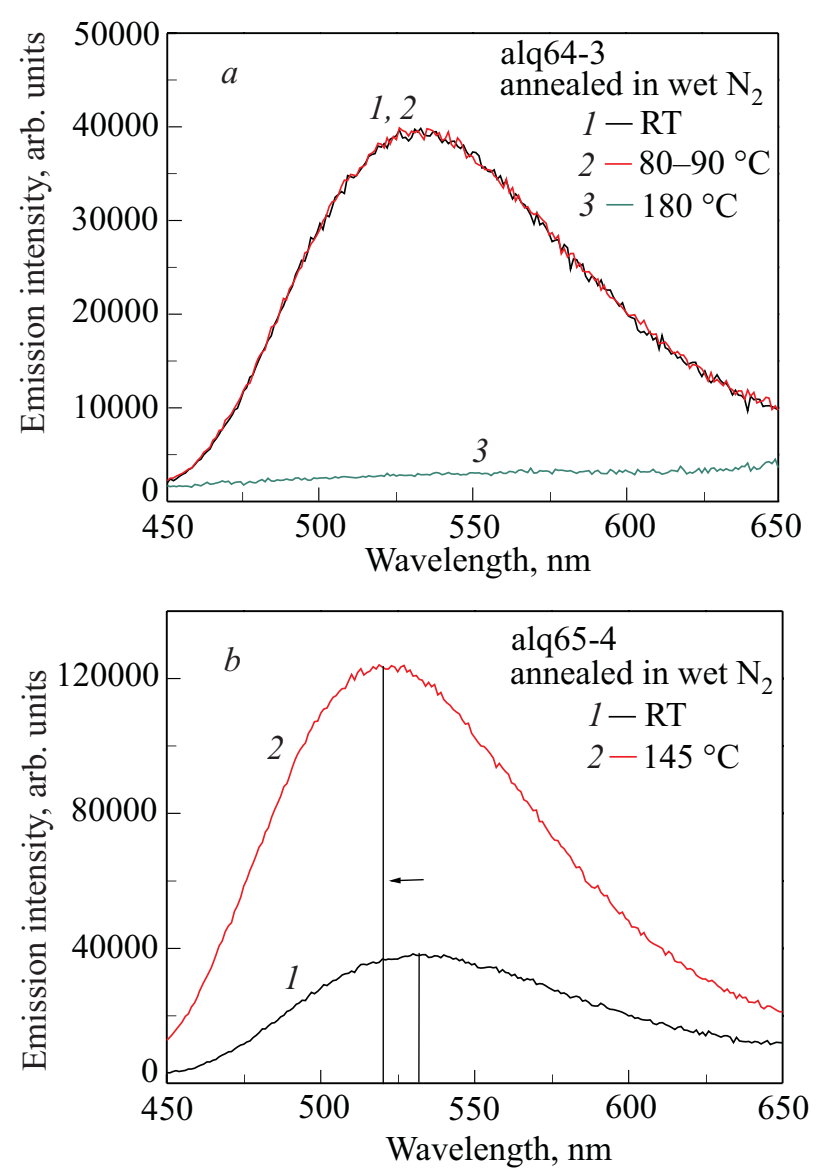

Fig. 5. (Color online) Emission bands of alq64-3 (a) and alq65-4 (b) samples during annealing treatment under wet nitrogen atmosphere.

sities of absorption and emission, which means that the observed absorptions in Figs. 1 and 3 do not correspond to the true absorptions which generate the observed emissions of Figs. 4 and 5,b. So, only the emission bands seem to describe more appropriately the changes which are happening in the $\mathrm{Alq}_{3}$ films during the annealing experiments, while the absorption bands are very likely the result of a

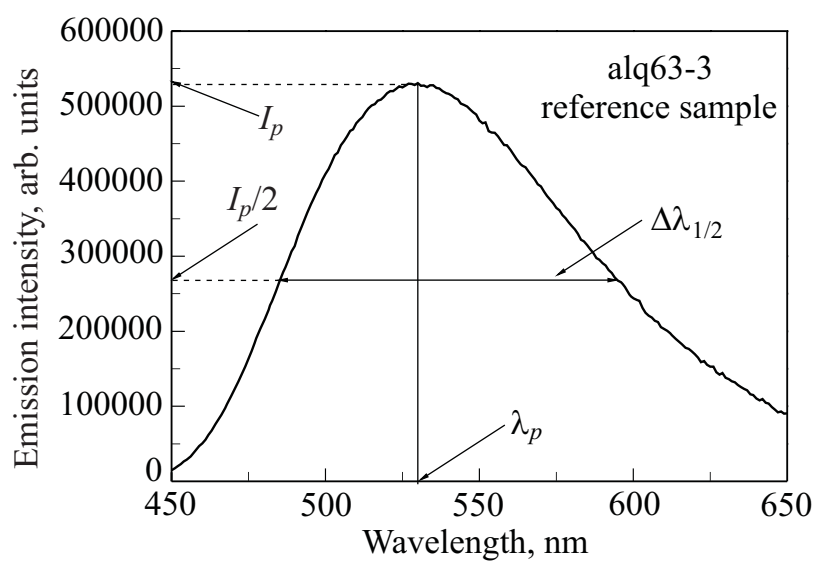

Fig. 6. (Color online) Emission band of sample alq63-3 and the main parameters which characterize its spectral features. The sample alq63-3 has not been annealed and is considered the reference sample. tangled sum of different bands which have not yet been resolved, and so they can only be used approximately to validate the emission measurements. Similar results have been obtained in all samples of Table 1, and it has been possible to measure accurately the peak intensity and wavelength variations of the emission bands during the annealing processes. In practice, the parameters which play a role during the annealing processes are reported in Fig. 6 on an emission band of the reference sample alq63-3. They are essentially the peak intensity, $I_{p}$, the wavelength of the emission peak, $\lambda_{p}$, and the linewidth at $1 / 2$ intensity, $\Delta \lambda_{1 / 2}$.

\section{Results and discussion}

In the previous section, it has been shown that annealing processes in different environment atmospheres lead to sizeable variations in the optical properties of thin films of pure $\mathrm{Alq}_{3}$ on glass substrates. Moreover, the experimental data collected are so numerous and significant that they deserve to be displayed in a meaningful way to extract as many as possibly useful parameters, also to understand what is going on in the films at molecular level. In the first place, it has been observed that, besides the temperature being the main agent, water is straight second in importance during the annealing processes. So, all data have been divided according to their origin from dry or wet atmospheres. Different gases as $\mathrm{N}_{2}, \mathrm{O}_{2}$ and air do not matter very much, apart a barely observable primacy of $\mathrm{O}_{2}$ which however is not enough to be taken in consideration in the present work.

Figure 7 shows the values of the peak intensity, $I_{p}$, measured at different temperatures and dry atmospheres. The values, normalized to those taken at RT, show that the emission remains unchanged up to $425 \mathrm{~K}$, increases to a maximum at about $480 \mathrm{~K}$, and practically disappears at $530 \mathrm{~K}$. Figure 8 shows the same items with reference to wet atmospheres. It is

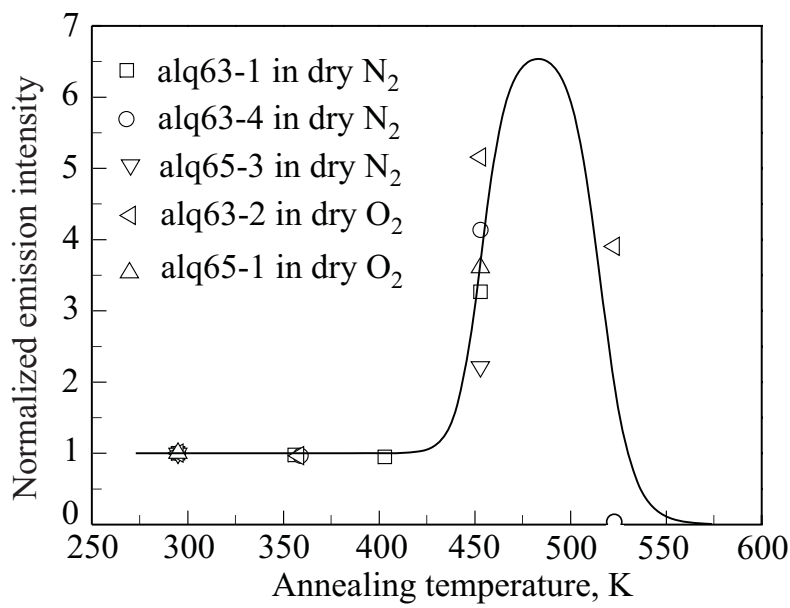

Fig. 7. Normalized peak intensities, $I_{p}$, of emission bands of five films of $\mathrm{Alq}_{3}$ annealed at each given temperature in dry oxygen and nitrogen, and best fit with two excitation energy processes, full line. See text for details. 


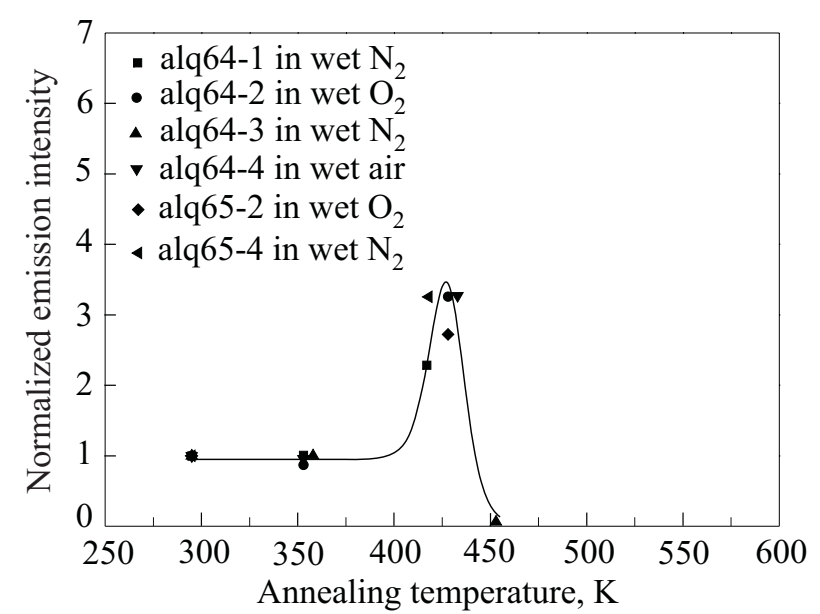

Fig. 8. (Color online) Normalized peak intensities, $I_{p}$, of emission bands of six films of $\mathrm{Alq}_{3}$ annealed at each given temperature in wet oxygen, nitrogen and air, and best fit with two excitation energy processes, full line. See text for details.

immediately seen that the intensity is lower, the increasing of the emission starts before, at $400 \mathrm{~K}$, and disappears much before, at $450 \mathrm{~K}$, than in the case of dry atmospheres, which is a further indication of the strong effect of water during the annealing processes. The full line which appears on both figures is an attempt to fit the experimental data which will be described in detail later on.

Figure 9 shows the wavelength of the emission peak, $\lambda_{p}$, measured at different temperatures and dry atmospheres. The experimental points display an abrupt decrease between 420 and $465 \mathrm{~K}$, while before and after they have constant and different values. Figure 10 shows the same items with reference to wet atmospheres, and the behavior is similar to the case of dry atmospheres with the difference that the abrupt decrease occurs well before, between 405 and $440 \mathrm{~K}$,

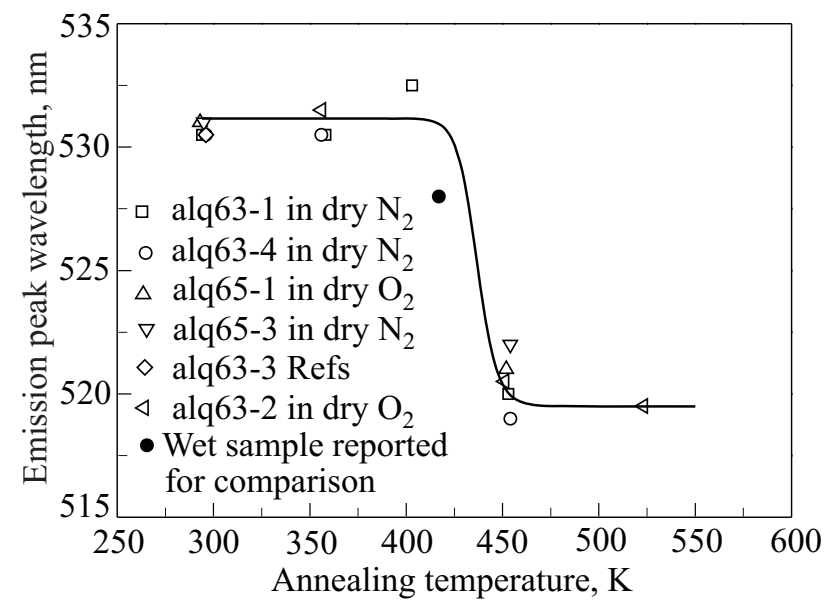

Fig. 9. Emission peak wavelength of $\mathrm{Alq}_{3}$ films annealed in dry atmosphere, and best fit with an activation process, full line. The full circle refers to sample alq64-1 annealed in wet $\mathrm{N}_{2}$ and has been reported for comparison.

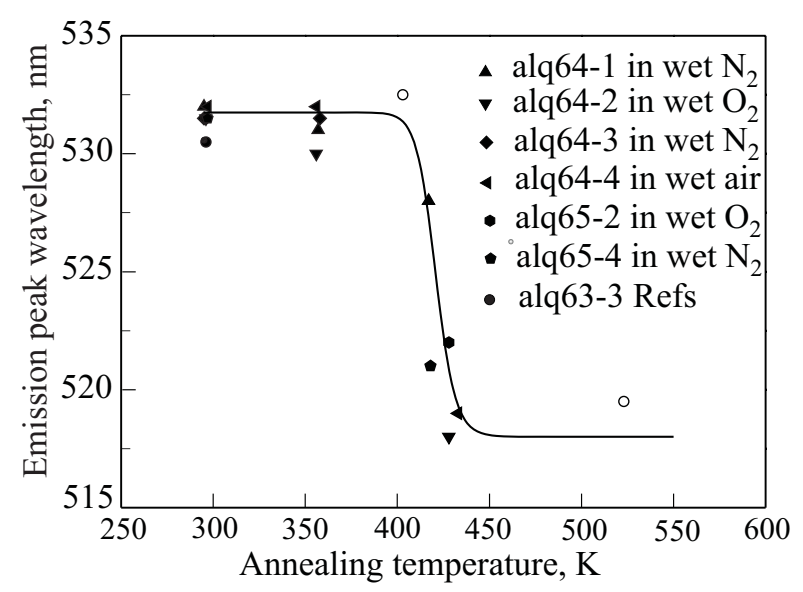

Fig. 10. Emission peak wavelength of $\mathrm{Alq}_{3}$ films annealed in wet atmospheres, and best fit with an activation energy process, full line. The empty circles refer to dry samples and are reported for comparison.

and furthermore the interval of temperature involved in the variation is smaller than before, $35 \mathrm{~K}$ against $45 \mathrm{~K}$. Moreover, the final blue shift is bigger in the wet samples with respect to the dry ones, about 14 against $12 \mathrm{~nm}$.

Figure 11 shows the linewidth, $\Delta \lambda_{1 / 2}$, of the emission band measured at different temperatures and dry atmospheres. The experimental points display an abrupt increase between 420 and $470 \mathrm{~K}$, while before and after they have constant and different values. Figure 12 shows the same items with reference to wet atmospheres, and the behavior is similar to the case of dry atmospheres with the difference that the abrupt increase occurs before, between 410 and $450 \mathrm{~K}$, and furthermore the interval of temperature involved in the variation is smaller than before, $40 \mathrm{~K}$ against $50 \mathrm{~K}$, somewhat similar to the case of the emission peaks. As for the blue shift, also the variation of linewidth seems to be bigger in wet samples than in dry ones.

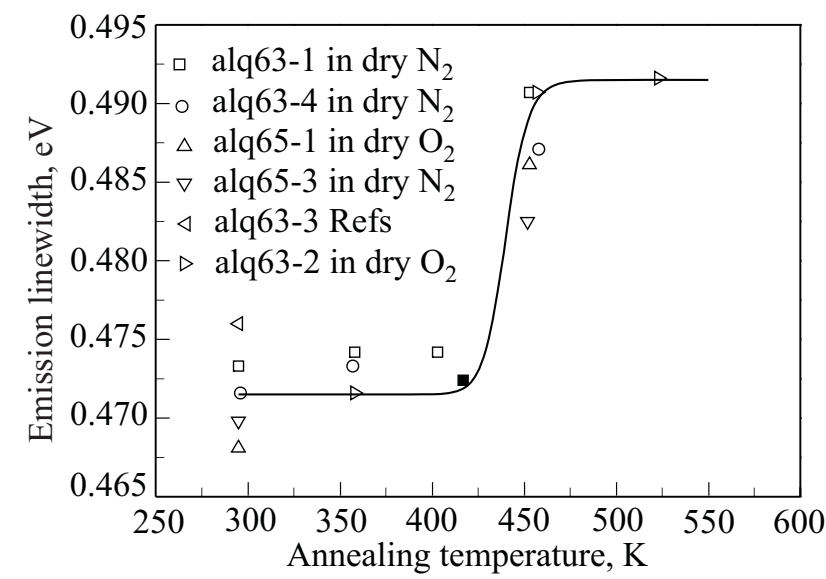

Fig. 11. Emission linewidth of $\mathrm{Alq}_{3}$ films annealed in dry atmospheres, and best fit with an activation process, full line. The full square refers to sample alq64-1 annealed in wet $\mathrm{N}_{2}$ and has been reported here for comparison. 


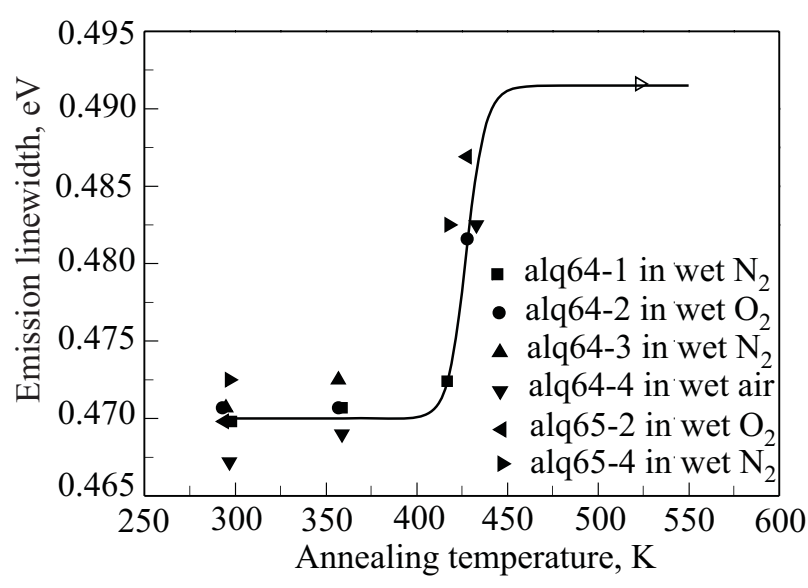

Fig. 12. Emission linewidth of $\mathrm{Alq}_{3}$ films annealed in wet atmospheres, and best fit with an activation energy process, full line. The empty triangle refers to sample alq63-2 annealed in dry $\mathrm{O}_{2}$ and has been reported here for comparison.

The experimental results reported in Figs. 7-12 have been interpreted as due to a phase transition first and to a chemical reaction later on, as the temperature increases. Activation energy processes are at work, and vapor adds a further parameter in both processes, in the sense that it promotes the phase transition at first, and accelerates the chemical reaction later on. Indeed, a close observation of the general features of the experimental data is providing some useful insights, as in the following.

As the temperature increases, the peak intensity, emission peak and linewidth start to change abruptly around $420 \mathrm{~K}$ in dry atmospheres, and around $405 \mathrm{~K}$ in wet atmospheres. This initial change looks like a phase transition which runs out in $40-50 \mathrm{~K}$, and is influenced by water which decreases the transition temperature, probably acting as a catalyzer. Once reached this new molecular state, the peak intensity of dry samples is practically zeroed at $530 \mathrm{~K}$, which corresponds to the destruction of the film itself. The same phenomenon occurs in wet samples, with the difference that the emission is zeroed at $450 \mathrm{~K}$, which means that water reacts with $\mathrm{Alq}_{3}$ molecules destroying the films much before than the temperature would have accomplished the same but alone.

However, both cases can be described by Arrheniustype phenomena where activation energies play a central role. By using the well-known expression for the Boltzmann distribution $\exp (-\Delta E / k T)$, where $\Delta E$ is the activation energy and $k T$ the thermal energy, and other known mathematical manipulations, it has been possible to simulate the experimental data reported in Figs. 7-12, and the full line in each of them is the obtained best fit. Table 2 reports the values of $T_{1 / 2}$, the temperature where the parameter under study increases or decreases by a factor 2 with respect to the whole variation, and $\Delta E$ is expressed in degrees Kelvin.

It is immediately observed that in dry samples the phase transition occurs at $440 \mathrm{~K}$, while the chemical reaction (in this case mainly evaporation) occurs at $510 \mathrm{~K}$, independently from the atmospheric gases. When vapor is added, the phase transition is anticipated to $420 \mathrm{~K}$, most probably water molecules act as promoters for the moving $\mathrm{Alq}_{3}$ molecules, and the chemical reaction (in this case a true chemical reaction) is also anticipated at $430 \mathrm{~K}$. The latter value of temperature is the main reason why samples in wet atmospheres do not reach high values of emission intensity, as it is instead the case of dry atmospheres. In other words, while at the beginning water seems to foster the molecular motion, soon after it starts to react with the same molecules destroying them very efficiently, and in any case more quickly than the temperature-assisted evaporation itself.

Different is the case of the activation energies, where the variations are much more contained. Indeed, while phase transitions possess a lower value of $\Delta E$ with respect to chemical reactions, which is expected, dry and wet atmospheres do not produce consistent variations, which was a surprise. Moreover, even more surprising is the average value of the energy activation among the various parameters, about $30,000 \mathrm{~K}(250 \mathrm{~kJ} / \mathrm{mol}, 2.5 \mathrm{eV})$, much bigger than $10 \mathrm{~kJ} / \mathrm{mol}$ as obtained by using a dynamic approach [9]. However, in the present work the simulation has been performed by using a steady state approach which very likely is not adequate in our circumstances. Indeed, by using a slightly different algorithmic, the value $\Delta E=1 \mathrm{eV}$ has been obtained recently for the blue shift [10]. Anyway, leaving aside for the moment the search of an exact theoretical explanation, the experimental facts reported in Figs. 7-12 describe abrupt changes of the optical properties of $\mathrm{Alq}_{3}$ films, which look like typical phase transitions.

Table 2. Half temperatures, $T_{1 / 2}$, and activation energies, $\Delta E$, in degrees Kelvin derived from the fits of the annealing measurements, see text for details. For convenience: $1,200 \mathrm{~K}=10 \mathrm{~kJ} / \mathrm{mol}=0.10 \mathrm{eV}$

\begin{tabular}{c|c|c|c|c|c}
\hline \multirow{2}{*}{$T_{1 / 2} / \Delta E(\mathrm{~K})$} & \multirow{2}{*}{ Gases } & \multicolumn{2}{|c|}{ Aggregation } & \multicolumn{2}{c}{ Reaction } \\
\cline { 3 - 5 } & & dry & wet & dry & $430 / 35,000$ \\
\hline$I_{p}$ & $\mathrm{~N}_{2}, \mathrm{O}_{2}$, air & $450 / 31,200$ & $420 / 30,074$ & $510 / 33,746$ & \\
$\lambda_{p}$ & $\mathrm{~N}_{2}, \mathrm{O}_{2}$, air & $435 / 36,529$ & $420 / 33,600$ & & \\
$\Delta \lambda_{1 / 2}$ & $\mathrm{~N}_{2}, \mathrm{O}_{2}$, air & $440 / 31,446$ & $425 / 35,000$ & & \\
Average & & $440 / 33,058$ & $420 / 32,891$ & $510 / 33,746$ & $430 / 35,000$ \\
\hline
\end{tabular}




\section{Conclusions}

Films of $\mathrm{Alq}_{3}$ are known to be amorphous up to $185^{\circ} \mathrm{C}$ $[11,12]$, so the phase transitions observed here consist of molecules of $\mathrm{Alq}_{3}$ which move towards new morphological aggregations, where they attain a blue shift and the emission efficiency is higher. This means that the intermolecular distances increase with the consequence of decreasing the reciprocal interactions, which leads to more efficient packings, as it has already been observed [11,13,14].

The most striking result of such phase transition is the sizeable increase of the emission at equal excitation after about $150{ }^{\circ} \mathrm{C}$, which is a paramount factor for application in luminous devices as OLEDs. Moreover, during annealing processes not only the emission is consistently increased, as shown here in Figs. 7 and 8, but also the lifetime of the emission attains much longer values $[5,6,15]$, which is another important factor for applications.

So, we are presently in a situation where appropriate annealing processes improve in a consistent way the luminous optical properties of $\mathrm{Alq}_{3}$ films, which is a much welcome result in itself, but still awaiting a reasonable explanation. In this regard, a brief attempt will be mentioned in the following.

Recently, by studying for very long times the PL of $\mathrm{Alq}_{3}$ films, it has been discovered that the emission itself is composed of four components which have their own spectral and time characteristics [16]. In particular, the fourth component possesses a blue shifted emission band with respect to the other three, and also a longer lifetime compared with them. As a matter of facts, at the beginning this component is also the less intense among the four one, so that the properties of as evaporated films are determined by the first three components, which are more red shifted and have short lifetimes. When the films are annealed, it happens that the fourth component becomes the dominant one, so that the emission of the film is blue shifted and the lifetime increases. In addition, as a bonus also the emission strength is increased, as shown in Figs. 7 and 8.

These emission components are supposed to belong to four molecular aggregations which pack in different way, but do not belong to the well-known crystalline phases of $\mathrm{Alq}_{3}$, although they may be some kind of precursors. These aggregations coalesce during the evaporation, and attain an equilibrium state among them depending on the rate of the evaporation and the temperature of the substrate, which explain the many different results obtained by various authors. When the films are subjected to annealing processes, higher temperatures supply the molecules with the energy needed to reach a more energetically convenient state of aggregation, which explains the occurrence of phase transitions, as described in the present work. It is interesting to note that, because the emission strength increases for all four aggregations, the molecular packing is moving to less dense states of matter, especially for the fourth aggregation which at the end results as the most optically efficient and long lived one $[6,15]$.

In conclusion, we are dealing in the present work with annealing processes which improve the optical properties of $\mathrm{Alq}_{3}$ films, a feat of tremendous importance to the growing field of optoelectronic applications, notably OLEDs. Moreover, it has been proposed that these new properties may be a consequence of morphological transformations which have been observed only by using the well-known and old technique of PL. Because the many modern microscopic investigative methods, among them atomic force microscopy (AFM), x-ray diffraction (XRD), $\mathrm{X}$-ray photoelectron spectroscopy (XRPS), Fourier transform infrared spectroscopy (FTIR), differential scanning calorimetry (DSC), and lately photocurrent spectroscopy (PS) [17], do not seem to be able to uncover yet the above aggregations, although their extreme utility in characterizing films of various materials, the PL method will be still pursued. In particular, because the previous hypothesis of the four molecular aggregations was born out of the four component model (FCM) for the emission [16], the PL of $\mathrm{Alq}_{3}$ films must be carefully studied in order to further validate the FCM, which is still being made.

Lately various authors have obtained results similar to those described in the present work in various molecules different from $\mathrm{Alq}_{3}[7,8,13,14]$, which leads to presume that also the properties of these molecules may be described with the FCM. However, it rests to future studies to establish whether the optical properties of many more molecular systems can be understood on the basis of a common phenomenological description, which may be the last but not the least issue of the optical luminous materials utilized in manufacturing OLEDs, and other optoelectronics devices.

\section{Acknowledgments}

The authors are indebted to T. Baldacchini and R.M. Montereali for useful discussions and suggestions. Many thanks are due to A. Pace for his earlier technical support.

1. C. Tang and S. Van Slyke, Appl. Phys. Lett. 51, 913 (1987).

2. M.J. Felton, Thinner Lighter Better Brighter, Today's Chemist at Work, American Chemical Society, November 2001, p. 31 .

3. Physics of Organic Semiconductors, W. Brütting (ed.), Wiley-VHC, Weinheim (2005).

4. K. Mullen and U. Scherf, Organic Light Emitting Devices: Synthesis, Properties, and Applications, K. Mullen and U. Scherf (eds.), Wiley-VCH, Verlag (2006).

5. G. Baldacchini, T. Baldacchini, P. Chiacchiaretta, R.B. Pode, and Q.-M. Wang, J. Lumin. 129, 1831 (2009).

6. G. Baldacchini, T. Baldacchini, P. Chiacchiaretta, R.B. Pode, M.A. Vincenti, and Q.-M. Wang, ECS Transactions 16(31), 3 (2009).

7. V.K. Shukla and S. Kumar, Synthetic Metals 160, 450 (2010). 
8. F.F. Muhammad and K. Sulaiman, Mater. Chem. Phys. 129 1152 (2011).

9. C.-P. Cho, C.-A. Wu, and T.-P. Perng, Adv. Funct. Mater. 16, 819 (2006).

10. G. Baldacchini, T. Baldacchini, P. Chiacchiaretta, R.B. Pode, and Q.-M. Wang, Effects of External Physical Parameters on Light Emission from $\mathrm{Alq}_{3}$ Films, in Frontier in Optics, Laser Science, Optical Fabrication and Testing and Organic Photonics and Electronics (2006), Optical Society of America, Washington, DC (2006), presentation number OPTuA3.

11. M. Brinkmann, G. Gadret, M. Muccini, C. Taliani, N. Masciocchi, and A. Sironi, J. Am. Chem. Soc. 122, 5147 (2000).

12. C.Y. Kwong, A.B. Djurisic, V.A.L. Roy, P.T. Lai, and W.K. Chan, Thin Solid Films 458, 281 (2004).
13. I. Hernandez, W.P. Gillin, and M. Somerton, J. Lumin. 129 1835 (2009).

14. I. Hernandez and W.P. Gillin, J. Phys. Chem. B2009, 113, 14079 (2009).

15. P. Chiacchiaretta, G. Baldacchini, T. Baldacchini, F. Bonfigli, R.B. Pode, R.M. Montereali, and M.A. Vincenti, Optical Properties, Morphology and Long Time Degradation of $\mathrm{Alq}_{3}$ Thin Films, Abstract Joint Meeting DGaO-SIOF, Brescia, 2-5 June 2009, DGaO-Proceedings 2009, http://www.dgao-proceedings.de — ISS:1614-8436, vol. 110, p. 28.

16. G. Baldacchini, T. Baldacchini, P. Chiacchiaretta, A. Pace, and R.B. Pode, J. Electrochem. Soc. 154, J217 (2007).

17. A. Scidà, Il Nuovo Cimento 34, 103 (2011). 